\title{
ON FUNDAMENTAL FUNCTIONS OF LAGRANGEAN INTERPOLATION
}

PAUL ERDÖS AND BÉLA A. LENGYEL

Introduction. It was shown in a number of recent papers that interpolation with fundamental abscissas chosen at the roots of various orthogonal polynomials is of considerable interest.

Let $I=[\alpha, \beta]$ be a closed interval on the real axis, and let $p(x)$ be greater than zero in $I$. The orthogonal polynomials with respect to $p(x)$ will be denoted by $\phi_{n}(x)$. Thus, by definition,

$$
\int_{\alpha}^{\beta} p(x) \phi_{n}(x) \phi_{m}(x) d x=\delta_{n m}, \quad n, m=0,1,2, \cdots .
$$

Erdös and Turán* proved important properties of the interpolating polynomial for the case when the zeros of $\phi_{n}(x)$ are taken for abscissas of interpolation. The theorems so deduced enabled the authors cited to draw important conclusions concerning the distribution of the roots of orthogonal polynomials. The proofs of Erdös and Turán are based on some properties of the fundamental functions of interpolation

$$
l_{k}^{n}(x)=\frac{\phi_{n}(x)}{\left(x-x_{k}^{n}\right) \phi_{n}^{\prime}\left(x_{k}^{n}\right)}, \dagger
$$

which we do not intend to repeat here. However, it is our purpose to add a few theorems concerning the properties of these fundamental functions. Our main result is the following theorem:

THEOREM. If $M \geqq p(x) \geqq m>0$, and if $p(x)$ is continuous in the finite interval $I=[\alpha, \beta]$ and the abscissas of interpolation are chosen as described above, then the maximum of $l_{k}^{n}(x)$ in $[\alpha+\epsilon, \beta-\epsilon]$ tends to one as $n$ tends to infinity for all $k$ for which $\alpha+\epsilon \leqq x_{k^{n}} \leqq \beta-\epsilon, \epsilon$ being an arbitrary positive number.

We shall make use of the following relations:

$$
\int_{\alpha}^{\beta} p(x) l_{i}^{n}(x) l_{k}^{n}(x) d x=0, \quad \text { if } \quad i \neq k,
$$

* On interpolation. I, Annals of Mathematics, (2), vol. 38 (1937), pp. 142-155.

$\dagger$ The function $l_{k}^{n}(x)$ is not the $n$th power of $l_{k}(x)$; it stands for $l_{k}^{(n)}(x)$. Similarly, $x_{k}{ }^{n}$ stands for $x_{k}^{(n)}$. The upper indices will be omitted completely in all formulas where there is no risk of misunderstanding. 


$$
\lambda_{k}^{n} \equiv \int_{\alpha}^{\beta} p(x) l_{k}^{n}(x) d x=\int_{\alpha}^{\beta} p(x)\left[l_{k}^{n}(x)\right]^{2}>0 .
$$

Erdös and Turán have proved that if $p(x) \geqq \delta>0$, then for any Riemann integrable function $f(x)$

where

$$
\lim _{n \rightarrow \infty} \int_{\alpha}^{\beta}\left[f(x)-L_{n}(f)\right]^{2} d x=0,
$$

$$
L_{n}(f) \equiv \sum_{i=1}^{n} f\left(x_{i}^{n}\right) l_{i}^{n}(x)
$$

Then from this theorem follows the well known property of $x_{i}{ }^{n}$ that the maximum of the distance of two consecutive abscissas tends to 0 as $n$ tends to infinity:

$$
\max _{i=1, \cdots, n}\left|x_{i}^{n}-x_{i-h}^{n}\right| \equiv \delta_{n} \rightarrow 0, \quad n \rightarrow \infty .^{*}
$$

For, if this were not true, then we could find a number $\delta>0$ and a sequence of integers $n_{1}, n_{2}, \cdots$ such that $\delta_{n_{k}} \geqq \delta>0$, for $k=1,2, \cdots$. The centers of the respective intervals which have the length $\delta_{n_{k}}$ would have at least one limit point $X$ in $I$. Thus there would exist a subsequence $m_{1}, m_{2}, \cdots$ such that the interval with the center $X$ and the length $\delta / 2$ would contain no roots of $\phi_{m_{k}}(x)$. The function $f(x)$, equal to one in this interval and zero elsewhere, is integrable, $L_{m_{k}}(f)=0$, and

$$
\int_{\alpha}^{\beta}\left[f-L_{m_{k}}(f)\right]^{2} d x=\frac{\delta}{2} \neq 0,
$$

in contradiction to the theorem. The same reasoning shows that $x_{1}^{n}-\alpha$ and $\beta-x_{n}^{n}$ also tend to zero with $1 / n$.

1. A minimum problem. We start with a well known minimum problem: Given $p(x) \geqq 0$, to determine the polynomial $f_{n}(x)$ of degree not exceeding $n-1$ subject to the condition $f(a)=1,(\alpha \leqq a \leqq \beta)$, which makes $\int_{\alpha}^{\beta} p(x)[f(x)]^{2} d x$ minimum. To solve this problem we write $f_{n}(x)=\sum_{t=1}^{n} a_{i} l_{i}(x)$, where the coefficients $a_{i}$ have to be determined. $\dagger$ The condition is

* This has been proved by Fejér for $p(x) \equiv 1$. His proof applies to the general case without any essential change. See also J. Shohat, Théorie générale des polynômes orthogonaux de Tchebichef, Mémorial des Sciences Mathématiques, vol. 66, p. 49. The dependence of $\delta$ on $n$ has been investigated by Erdös and Turán, On interpola. tion, II, Annals of Mathematics, (2), vol. 39 (1938), p. 702.

$\dagger$ Cf. the second footnote. 


$$
f_{n}(a)=\sum_{i=1}^{n} a_{i} l_{i}(a)=1,
$$

and the integral which has to be minimized is

$$
\int_{\alpha}^{\beta} p(x)\left[f_{n}(x)\right]^{2} d x=\int_{\alpha}^{\beta} p(x) \sum_{i=1}^{n} \sum_{j=1}^{n} a_{i} a_{j} l_{i}(x) l_{j}(x) d x .
$$

In consequence of equations (1) and (2) this becomes

$$
\sum_{i=1}^{n} \lambda_{i} a_{i}^{2}
$$

If we consider the quantities $a_{i} \lambda_{i}{ }^{1 / 2}$ and $l_{i}(a) / \lambda_{i}{ }^{1 / 2}$ as components of vectors in an $n$-dimensional space, then it becomes evident that (4) attains its minimum if $a_{i} \lambda_{i}{ }^{1 / 2}=C l_{i}(a) / \lambda_{i}{ }^{1 / 2}$, where

$$
C=\frac{1}{\sum_{i=1}^{n} \frac{\left[l_{i}(a)\right]^{2}}{\lambda_{i}}}
$$

Thus we find*

$$
f_{n}(x)=\frac{1}{\sum_{i=1}^{n} \frac{\left[l_{i}(a)\right]^{2}}{\lambda_{i}}} \sum_{i=1}^{n} \frac{l_{i}(a) l_{i}(x)}{\lambda_{i}}
$$

In the special case when $a$ coincides with a root of $\phi_{n}(x)$, say $x_{k}{ }^{n}$, we have $f_{n}(x) \equiv l_{k}^{n}(x)$; that is, the fundamental function is the minimizing function. This can be verified directly.

Let $A$ and $B$ be positive numbers. The polynomial $f_{n}(x)$ of degree less than $n$, which has the value $A$ at the point $x_{k}$ and the value $B$ at the point $x_{r}$ and minimizes the integral of $p(x)[f(x)]^{2}$ over $I$, is $A l_{k}(x)+B l_{r}(x)$. In fact, let

$$
f_{n}(x)=\sum_{i=1}^{n} a_{i} l_{i}(x)
$$

The two conditions are

* The same solution is given in the theory of orthogonal polynomials in the form $f_{n}(x)=\sum_{i=0}^{n-1} \phi_{i}(x) \phi_{i}(a) / \sum_{i=0}^{n} \phi_{i}{ }^{2}(a)$. Comparing with equation (5) we get $\sum_{i=0}^{n} \phi_{i}{ }^{2}(a)=\sum_{i=1}^{n} l_{i}{ }^{2}(a) / \lambda_{i}$, and this evidently holds for any $a$ (both sides being polynomials). Cf. J. Shohat, On interpolation, Annals of Mathematics, (2), vol. 34 (1933), pp. 130-146; p. 145. 


$$
\sum_{i=1}^{n} a_{i} l_{i}\left(x_{k}\right)=a_{k}=A, \quad \sum_{i=1}^{n} a_{i} l_{i}\left(x_{r}\right)=a_{r}=B .
$$

The sum $\sum_{l=1}^{n} a_{i}{ }^{2} \lambda_{i}$ has to be a minimum. Evidently $a_{i}=0$ if $i \neq k$, and $i \neq r$.

2. Bounds of the fundamental functions. It was supposed in $\$ 1$ that $p(x) \geqq 0$. From now on we shall suppose that $p(x)$ is continuous in $I$ and remains between two fixed positive bounds, $M \geqq t(x) \geqq m>0$.

Let $f_{n}(x)_{a}$, or briefly $f_{n}(x)$, denote the polynomial described at the beginning of $\$ 1$, that is, the polynomial of degree $n-1$ which minimizes the integral of $p(x)[f(x)]^{2}$ over $I$ under the restriction $f(a)=1$. The subscript $a$ is applied to indicate the dependence of $f_{n}$ on $a$.

THEOREM 1. If $a$ is in the interior of $I$, then to every $\delta>0$ there corresponds an $\epsilon$ such that

$$
f_{n}(x)_{a}<1+\delta
$$

for all $n$, whenever $|x-a|<\epsilon$.

If $a$ is restricted to any closed subinterval of $I$, then there exists an $\epsilon$ independent of a such that (6) holds if $|x-a|<\epsilon$.

If the theorem were not true, then for given $a$ and any small $\epsilon>0$ there would exist at least one $n$ such that for some $\xi_{n}$

$$
f_{n}\left(\xi_{n}\right)=1+\delta
$$

and $\alpha \leqq a-\epsilon \leqq \xi_{n} \leqq a+\epsilon \leqq \beta$. Here we use the fact that $a$ is in the interior of $I$. Without loss of generality we can assume that $\alpha=0$ and that $\xi_{n}<a$. We introduce the function $g_{n}(x)=f_{n}\left(c_{n} x\right) /(1+\delta)$, where $c_{n}=\xi_{n} / a<1$. Evidently $g_{n}(a)=f_{n}\left(\xi_{n}\right) /(1+\delta)=1$; hence

$$
\int_{\alpha}^{\beta} p(x)\left[g_{n}(x)\right]^{2} d x \geqq \int_{\alpha}^{\beta} p(x)\left[f_{n}(x)\right]^{2} d x .
$$

On the other hand, setting $y=c_{n} x$, we obtain*

$$
\begin{aligned}
\int_{0}^{\beta} p(x)\left[g_{n}(x)\right]^{2} d x & =\frac{1}{c_{n}(1+\delta)^{2}} \int_{0}^{c_{n} \beta} p\left(y / c_{n}\right)\left[f_{n}(y)\right]^{2} d y \\
& <\frac{1}{c_{n}(1+\delta)^{2}} \int_{0}^{\beta} p(x / c)\left[f_{n}(x)\right]^{2} d x
\end{aligned}
$$

By hypothesis, $0<a-\xi_{n}=a\left(1-c_{n}\right)<\epsilon, 0<1-c_{n}<\epsilon / a$. Hence if $\delta$ and $a$ are fixed, then $\epsilon$ (independent of $n$ ) can be chosen so small that

\footnotetext{
${ }^{*}$ For $x>\beta$ we define $p(x)=p(\beta)$.
} 
$c_{n}(1+\delta)>1$. Thus by (8) and (9) we have the following inequality:

$$
(1+\delta) \int_{0}^{\beta} p(x)\left[f_{n}(x)\right]^{2} d x<\int_{0}^{\beta} p\left(x / c_{n}\right)\left[f_{n}(x)\right]^{2} d x
$$

or

$$
\delta \int_{0}^{\beta} p(x)\left[f_{n}(x)\right]^{2} d x<\int_{0}^{\beta}\left[p\left(x / c_{n}\right)-p(x)\right]\left[f_{n}(x)\right]^{2} d x .
$$

However, this last inequality contains a contradiction. For, if $\epsilon$ is small enough, then on account of the continuity of $p(x)$,

$$
\left|p(x)-p\left(x / c_{n}\right)\right|<\delta m
$$

for all $c_{n}$ such that $1>c_{n}>1-\epsilon / a$. Hence (11) leads to a contradiction, namely

$$
\delta \int_{0}^{\beta} p(x)\left[f_{n}(x)\right]^{2} d x<\delta m \int_{0}^{\beta}\left[f_{n}(x)\right]^{2} d x<\delta \int_{0}^{\beta} p(x)\left[f_{n}(x)\right]^{2} d x .
$$

This proof depends on the possibility of making $1-c_{n}$ small by choosing $\epsilon$ small enough. This was possible for every fixed $a$ because of the relation $0<1-c_{n}<\epsilon / a$. Of course $\epsilon$ depends on $a$. However, if $a$ is confined to a closed subinterval $I^{\prime}$, wholly in the interior of $[0, \beta]$, then there exists an $\epsilon$ for which (6) holds for all $a$ throughout $I^{\prime}$. Thereby Theorem 1 is proved.

THEOREM 2. Let $I^{\prime}$ be any closed subinterval wholly in the interior of $I$, and let $\epsilon$ be a fixed positive number; then for all $k$ for which $x_{k}^{n}$ is in $I^{\prime}$ the maximum of $l_{k}^{n}(x)$ in the set consisting of $I^{\prime}$ minus the interval $\left[x_{k}^{n}-\epsilon, x_{k}^{n}+\epsilon\right]$ tends uniformly to 0 as $n$ tends to infinity.

The word "uniformly" has to be understood as follows: Let $A_{n k}$ be the maximum of $l_{k}^{n}(x)$ in the set described in the theorem; then for every $\eta>0$ there exists an $N$ such that $A_{n k}<\eta$ whenever $n>N$ for all $k$ such that $x_{k}^{n}$ is contained in $I^{\prime}$. This theorem states the fact that the fundamental functions $l_{k}^{n}(x)$ which belong to abscissas entirely in the interior of $I$ are small everywhere, except possibly at both ends of the interval $I$ and in a small neighborhood of the respective abscissas to which they belong.

Proof. Let $I^{\prime \prime}$ denote the difference of the set $I^{\prime}$ and the open interval $\left[x_{k}^{n}-\epsilon, x_{k}^{n}+\epsilon\right]$. The interval $I^{\prime \prime}$ will depend on $\epsilon, n$, and $k$; it will consist of two intervals $\left[\alpha^{\prime}, x_{k}^{n}-\epsilon\right]$ and $\left[x_{k}^{n}+\epsilon, \beta^{\prime}\right]$. Let $\xi$ be the abscissa in $I^{\prime \prime}$ for which $l_{k}^{n}(\xi)=A_{n k}$. Evidently $\xi$ cannot coincide with any root $x_{i}$, for $l_{k}^{n}\left(x_{i}\right)=0$ if $i \neq k$. We may assume that $x_{k}^{n}=0$, 
since we can always introduce, if necessary, a new variable $x^{\prime}=x-x_{k}^{n}$. We may also assume that $\xi \geqq x_{k}^{n}+\epsilon=\epsilon$.

If $n$ is large enough, the roots of $\phi_{n}(x)$ extend beyond both ends of the interval $I^{\prime}$, in consequence of the fact explained in the last paragraph of the introduction. Therefore $\xi$ lies between two roots of $\phi_{n}(x)$ :

$$
x_{r-1}<\xi<x_{r} .
$$

Let $c=\xi / c_{r}<1$. (It will be well to remember that $x_{r}, \xi$, and $c$ depend on $n$, and that we use them as abbreviations for $x_{r}^{n}, \xi_{n}$, and $c_{n}$.) The polynomial $f(x)=l_{k}^{n}(c x)$ has the following properties:

(i) It is of degree $n-1$.

(ii) $f(0)=l_{k}^{n}(0)=1$.

(iii) $f\left(x_{r}\right)=l_{k}^{n}\left(c x_{r}\right)=A_{n k}$.

It follows from the results of $\$ 1$ that

$$
\begin{aligned}
\int_{\alpha}^{\beta} p(x)\left[l_{k}(c x)\right]^{2} d x & \geqq \int_{\alpha}^{\beta} p(x)\left[l_{k}(x)+A_{n k} l_{r}(x)\right]^{2} d x \\
& =\lambda_{k}+A_{n k} \lambda_{r} .
\end{aligned}
$$

Substituting $y=c x$, we obtain

$$
\begin{aligned}
\int_{\alpha}^{\beta} p(x)\left[l_{k}(c x)\right]^{2} d x & =\frac{1}{c} \int_{\alpha c}^{\beta c} p(y / c)\left[l_{k}(y)\right]^{2} d y \\
& <\frac{1}{c} \int_{\alpha}^{\beta} p(x / c)\left[l_{k}(x)\right]^{2} d x .
\end{aligned}
$$

According to the last paragraph of the introduction, the maximum of $\left|x_{i}^{n}-x_{i}^{n}-1\right|$ tends to 0 with $1 / n$. Moreover, by hypothesis $\xi>\epsilon$; therefore, given any $q<1$, there exists an $N$ such that, by virtue of (12), $q<\xi_{n} / x_{n}=c_{n}<1$ for all $n>N$. On account of the continuity of $p(x)$, for any $\eta>0$ there exists a $q$ such that $|p(x)-p(x / c)|<\eta m$, if $q<c<1$. Hence

$$
\begin{aligned}
\int_{\alpha}^{\beta}[p(x)-p(x / c)]\left[l_{k}^{n}(x)\right]^{2} d x & <\eta m \int_{\alpha}^{\beta}\left[l_{k}^{n}(x)\right]^{2} d x \\
& \leqq \eta \int_{\alpha}^{\beta} p(x)\left[l_{k}^{n}(x)\right]^{2} d x=\eta \lambda_{k} .
\end{aligned}
$$

We now obtain from equations (13), (14), and (15) the relation

$$
\lambda_{k}+A_{n k} \lambda_{r}<\frac{1}{c} \lambda_{k}(1+\eta)=\lambda_{k}+\lambda_{k}\left(\frac{1-c}{c}+\frac{1}{c} \eta\right) .
$$


Here $1-c$ and $\eta$ can be chosen arbitrarily small provided that $n$ is large enough. Thus, if we write out the indices $n$ and divide by $\lambda_{r}>0$,

$$
A_{n k}<\frac{\lambda_{k}^{n}}{\lambda_{r}^{n}} \epsilon_{n},
$$

where $\epsilon_{n}$ tends to 0 with $1 / n$. In concluding the proof of the theorem we shall prove the following lemma:

Lemma. If the conditions of Theorem 2 are satisfied, then there exists a positive constant $K$ (independent of $n$ ) such that $\lambda_{i}{ }^{n} / \lambda_{k}{ }^{n} \leqq K$, for all $x_{i}^{n}$ and $x_{k}^{n}$ in $I^{\prime}$.

In other words, the generalized Cotes' numbers belonging to abscissas which do not come too close to the ends of the interval $I$ are of the same order of magnitude.

We may assume that $I$ is $[0,1]$ and that $x_{k}<x_{i}$. If $\lambda_{k} \geqq \lambda_{i}$, the statement in the lemma is evident with $K=1$. If $\lambda_{k}<\lambda_{i}$, we introduce the notation $c=x_{k} / x_{i}<1$ and $y=c x$. By reasoning similar to that used in the proof of the theorem we obtain

$$
\begin{aligned}
& \int_{0}^{1} p(x)\left[l_{k}(c x)\right]^{2} d x>\lambda_{i}, \\
& \int_{0}^{1} p(x)\left[l_{k}(c x)\right]^{2} d x<\frac{M}{c m} \lambda_{k} .
\end{aligned}
$$

By hypothesis $x_{k} \geqq \alpha^{\prime}>0$ and $x_{i}<1$, therefore $c>\alpha^{\prime}$ and

$$
\lambda_{i}{ }^{n}<\frac{1 M}{\alpha^{\prime} m} \lambda_{k^{n}} \equiv K \lambda_{k}{ }^{n}
$$

which we were to prove.

THEOREM 3. If $I^{\prime}$ is any closed interval wholly in the interior of $I$, then the maximum of $l_{k}^{n}(x)$ in $I^{\prime}$ tends to 1 as $n$ tends to infinity for all $x_{k}{ }^{n}$ in $I^{\prime}$.

For if any $\delta>0$ is given, then, according to Theorem 1 , we can find $\epsilon>0$ such that for any abscissa $x_{k}{ }^{n}$ in $I^{\prime}, l_{k}^{n}(x)<1+\delta$ whenever $\left|x-x_{k}^{n}\right|<\epsilon$. Outside this interval, that is, in $\left[\alpha^{\prime}, x_{k}^{n}-\epsilon\right]$ and in $\left[x+\epsilon, \beta^{\prime}\right]$, the maximum of $l_{k}^{n}(x)$ tends to 0 with $1 / n$ by Theorem 2 ; therefore there exists an $N$ such that the maximum of $l_{k}^{n}(x)$ in $I^{\prime \prime}$ is less than one whenever $n>N$. Finally, $l_{k}^{n}\left(x_{k}^{n}\right)=1$.

Victoria University OF MANCHESTER AND

WORCESTER, MASS. 